\title{
Occurrence and faecal shedding of extended-spectrum beta-lactamase-producing Escherichia coli in sows and their offspring
}

\author{
Jan Vašek ${ }^{1}$, Jonáš Vaňhara ${ }^{1}$, Monika Dolejskáe, ${ }^{2,3}$, Martina Masař́íková4, \\ Alois Ćížek ${ }^{3,4}$, Jiří Smola ${ }^{1,3}$ \\ University of Veterinary and Pharmaceutical Sciences Brno, ${ }^{1}$ Faculty of Veterinary Medicine, Ruminant and \\ Swine Clinic, ${ }^{2}$ Faculty of Veterinary Hygiene and Ecology, Department of Biology and Wildlife Diseases, \\ ${ }^{3}$ CEITEC VFU, ${ }^{4}$ Faculty of Veterinary Medicine, Department of Infectious Diseases \\ and Microbiology, Brno, Czech Republic
}

Received April 22, 2020

Accepted June 22, 2020

\begin{abstract}
The aim of the present study was to monitor the presence of extended-spectrum beta-lactamase (ESBL) producing E. coli on farm A with the history of previous use of ceftiofur in suckling pigletsand to analyse the risk factors of selection and dissemination of ESBL producers in the production herd. In the year of 2014, a total of 411 samples (rectal swabs or faeces)from pigs of various age categories (sows, gilts and suckling piglets) were collected. The sampling was performed more than 24 months after the ban of ceftiofur on the farm. The sows and gilts were sampled repeatedly before and after farrowing. All collected samples were directly cultivated on MacConkey agar (MCA) containing cefotaxime $(2 \mathrm{mg} / \mathrm{l})$ and obtained sub-cultures were tested for ESBL production by double disc synergy test. According to our results, all gilts were negative for ESBL-producing E. coli in the introduction period, however, the excretion of ESBL-producing E. coli was observed before and after delivery. Most of the new-born piglets from positive sows and gilts shed ESBL-producing E. coli early after birth. All tested ESBL-producing isolates were resistant to multiple antimicrobials, suggesting that antibiotics from other groups used for therapy co-select for ESBL producers in pigs on the studied farm. Intestinal colonization of lactating sows and their offspring as well as survival of ESBL-producing E. coli in the farm environment should be recognised as important risk factors of circulation and long-time persistence of ESBL producers in the herd.
\end{abstract}

Ceftiofur, new-born piglets, persistence, ESBL

Antimicrobial agents used for therapy and prophylaxis of bacterial infections in foodproducing animals constitute the risk for selection of antimicrobial resistance in enteric commensal bacteria. Escherichia coli is a dominant part of normal intestinal flora in humans and animals as well as an important pathogen causing various infections. It also represents an important reservoir of antibiotic resistance determinants. The increasing prevalence of E. coli resistant to major antimicrobials in food-producing animals has become global public health concern (Seiffert et al. 2013).

Extended-spectrum cephalosporins, especially those of the $3^{\text {rd }}$ and $4^{\text {th }}$ generation, are among the most important used antimicrobial agents in human and veterinary medicine. Cephalosporins are widely prescribed against various enteric infections in farm and pet animals (Hornish and Kotarski 2002). The resistance of enteric bacteria totheir antimicrobials is mostly caused by the bacterial production of extended-spectrum betalactamases (ESBL) or AmpC beta-lactamases (Smet et al. 2010). These enzymes are classified into several groups, among which CTX-M enzymes are the most prevalent, successively disseminating in animals and humans all over the world (D'Andrea et al. 2013). ESBL-producing organisms exhibit resistance to multiple antibiotics apart from beta-lactams, thus the antimicrobial agents of various groups used for the therapy are able to co-select the ESBL producers (Pitout and Laupland 2008).

Address for correspondence:

Jan Vašek

Ruminant and Swine Clinic, Faculty of Veterinary Medicine

University of Veterinary and Pharmaceutical Sciences Brno

Palackého tř. 1946/1, 61242 Brno, Czech Republic 
ESBL-producing E. coli isolates have been increasingly documented in pig farms in Europe (EFSA 2011). One of the main factors for the selection and spread of ESBLproducing $E$. coli among food-producing animals is the excessive use of cephalosporins. Several studies proved a link between the use of $3^{\text {rd }}$ and $4^{\text {th }}$ generation cephalosporins and selection of ESBL-producing E. coli in poultry (Dutil et al. 2010), cows (Tragesser et al. 2006; Dolejska et al. 2011) and pigs (Jørgensen et al. 2007; Lutz et al. 2011; Agersø et al. 2012; Andersen et al. 2015). The occurrence of ESBL-producing commensal E. coli in swine is alarming due to the potential risk of transfer of antibiotic resistance determinants to human microbiome via the food chain (Denkel et al. 2016). Increasing prevalence of ESBL-producing $E$. coli led to a voluntary ban or significant reduction of cephalosporin use in pig production in some European countries (EMA 2015).

The aim of the study was to evaluate the circulation of ESBL-producing $E$. coli isolates in sows and their piglets on farm A two years after a voluntary withdrawal of ceftiofur that had been used as part of the control program against Streptococcus suis in sucking piglets, and to determine the occurrence of ESBL-producing E. coli in gilts originating from a multiplier herd on farm B where cephalosporins had never been used. The colonization by ESBL producers was monitored before and after their introduction into the herd on farm A.

\section{Materials and Methods}

Farm description, study design and sampling

Farm A is a conventional farm with one site operation housing 1300 sows in 2014 . The use of ceftiofur for the metaphylactic purposes was banned on the farm in December 2012, more than 24 months before this study was conducted. Antimicrobials used on the farm included tiamulin, amoxicillin and a combined therapy by macrolides (tilmicosin, aivlosin) and chlortetracycline.

Rectal swabs or faeces were taken from sows, gilts and piglets. All samples were refrigerated and analysed in the laboratory within $24 \mathrm{~h}$.

Rectal swabs from a total of 100 pregnant sows of second and higher parity (farm A) were taken a few days before farrowing. For sows that were positive for ESBL-producing E. coli, samples were also taken after the farrowing or after 7 days. For that purpose, faeces samples were collected in order to quantify the excretion of ESBL-producing E. coli. Samples were taken from litters of the positive sows; in total, rectal swabs of 72 piglets aged one to eight days were examined.

Gilts originating from a multiplier herd at farm B with no history of cephalosporins were sampled before and after their introduction into the sow herd at farm A. Rectal swabs from 110 gilts during the isolation period on farm A were collected. Thirty-five randomly selected pregnant gilts were sampled approximately 107 days after their introduction to the herd, a few days before farrowing. Gilts that were found positive for ESBL-producing E. coli were sampled also after the farrowing and tested for excretion of ESBL-producing E. coli. Rectal swabs were taken from 55 randomly selected one to seven-days old suckling piglets of the positive gilts.

\section{Isolation of ESBL-producing E. coli}

The samples of rectal swabs were cultivated directly on MacConkey agar (Oxoid, Basingstoke, UK) supplemented with cefotaxime (Sigma-Aldrich, Prague, Czech Republic) at a concentration of $2 \mathrm{mg} / 1$ (MCA $\mathrm{CEFF}$ ). Species identification of one presumptive colony per sample per bacteria of interest was made using the Matrixassisted laser desorption/ionization time of flight (MALDI-TOF) system (Bruker Daltonics, Brehmen, Germany). Mass spectra were estimated using the software FlexControl-microflex v 3.3 (Bruker Daltonics, Brehmen, Germany) and MaldiBiotyper v 3.0 (Bruker Daltonics, Brehmen, Germany). The resistant colonies identified as E. coli were tested for production of ESBL using double-disc synergy test (CLSI 2008). The ESBL-producing strain E. coli $172 \mathrm{DI} / \mathrm{B}$ was used as a positive control.

Faeces samples from sows and gilts were first processed semi-quantitatively to estimate the counts of cefotaxime-resistant coliform. They were inoculated on $\mathrm{MCA}_{\mathrm{CEF}}$ using a 1- $\mu$ l disposable plastic loop by the streak plate method into four quadrants. The loop was discarded between each sector. Quantification was expressed as $1+, 2+, 3+, 4+$ or $5+$ based on the number of quadrants that demonstrated bacterial growth. Growth of a few isolated colonies limited to quadrant 1 was categorized as $1+$, growth of many colonies limited to quadrant1 was categorized as 2+, growth limited to quadrants 1 and 2 was categorized as 3+, growth in quadrants 1, 2 and 3 was categorized as $4+$ and bacterial growth that extended to all 4 quadrants as 5+ (Van Saene et al. 2012). Viable counts of cefotaxime-resistant coliforms in randomly selected faeces samples were determined. First, $1 \mathrm{~g}$ of faeces was diluted $10 \times$ in buffered peptone water, followed by homogenization of the sample by vigorous vortexing. These $10^{-1}$ homogenates were again diluted $10 \times\left(10^{-2}\right.$ dilution $)$ and $100 \times\left(10^{-3}\right.$ dilution $)$ using peptone saline, 
and $100 \mu \mathrm{l}$ of all three dilutions were streaked on $\mathrm{MCA}_{\mathrm{CEF}} \cdot$ Cefotaxime-resistant lactose-positive colonies were counted to determine mean bacterial densities expressed as $\mathrm{CFU} / \mathrm{g}$.

Antimicrobial susceptibility testing

Selected ESBL-producing E. coli isolates were further tested for antimicrobial susceptibility using the disk diffusion method and the following discs according to CLSI criteria (CLSI 2008): amoxicillin/clavulanic acid (AMC; $30 \mu \mathrm{g}$ ), ampicillin (AMP; $10 \mu \mathrm{g}$ ), cefoxitin (FOX; $30 \mu \mathrm{g})$, ceftazidime (CAZ; $30 \mu \mathrm{g})$, cephalothin (CEP; $30 \mu \mathrm{g}$ ), chloramphenicol (CHL; $30 \mu \mathrm{g}$ ), ciprofloxacin (CIP; $5 \mu \mathrm{g}$ ), gentamicin (GEN; $10 \mu \mathrm{g}$ ), imipenem (IMP; $10 \mu \mathrm{g}$ ), nalidixic acid (NA; $30 \mu \mathrm{g})$, streptomycin (STR; $10 \mu \mathrm{g}$ ), trimethoprim-sulphamethoxazole (SXT; $25 \mu \mathrm{g}$ ), compound sulphonamides (S3; $300 \mu \mathrm{g}$ ) and tetracycline (TCY; $30 \mu \mathrm{g}$ ) (Oxoid, Basingstoke, UK). We used CLSI susceptibility testing guideline breakpoints to categorize isolates into susceptible and resistant category (CLSI 2008).Escherichia coli ATCC25922 (American Type Culture Collection) was used for quality control of the disk diffusion method.

\section{Results}

ESBL-producing E. coli isolates were found in rectal swabs of $25(25 \%, \mathrm{n}=100)$ pregnant sows. The sows that were found positive were sampled repeatedly shortly after the delivery and ESBL-producing E. coli isolates were identified in $96 \%$ animals in counts ranging from $10^{1}$ to $10^{6} \mathrm{CFU}$ (mean $10^{3}$ ) per gram of faeces. Litters of 16 positive sows were sampled and ESBL-producing $E$. coli isolates were found in 15 of them (Table 1). Faeces samples were collected from three to 11 piglets per litter, in total 72 piglets aged one to seven days were examined. ESBL producers were isolated from $45(63 \%, n=72)$ new-born piglets. Eight litters were excluded from the study because of the presence of cross-fostering piglets.

Gilts (farm B with no history of cephalosporins) examined during the isolation period did not carry ESBL producers. However, during the second sampling shortly before the farrowing and approximately three months after their introduction into the herdon farm A, ESBL-producing E. coli isolates were found in $12(34 \%, \mathrm{n}=35)$ animals. Most gilts (11 out of 12) excreted ESBL producers in counts ranging from $10^{3}$ to $10^{5} \mathrm{CFU}$ (mean $10^{5}$ ) per gram of faeces (Table 1). ESBL producers were detected in all but one litters of the positive gilts. Five to seven piglets per litter were examined and a total of $42(76 \%$, $\mathrm{n}=55)$ piglets carried ESBL-producing E. coli. Two litters were excluded because of cross-fostering piglets.

The results of antibiotic susceptibility testing of 107 randomly selected ESBLproducing E. coli are summarized in Table 2. Resistance to ampicillin and cephalotin associated with ESBL phenotype was found in all isolates. Majority of the isolates $(94.3 \%)$ showed resistance to streptomycin followed by resistance to sulphonamides $(82.2 \%)$, tetracyclines $(80.4 \%)$, trimethoprim-sulphamethoxazole $(53.3 \%)$ and chloramphenicol (45.8\%). Resistance to amoxicillin/clavulanic acid, cephalosporins (cefoxitin, ceftazidime, cephalothin), ciprofloxacin, gentamicin and imipenem was not found. All isolates showed multi-resistance profile defined as resistance to two and more substances from different antimicrobial groups. Resistance to four antimicrobial agents including streptomycin, sulphonamides, tetracycline and chloramphenicol was the most common type identified in $23.3 \%$ isolates. The second most prevalent phenotype was resistance to substances from five antimicrobial groups (streptomycin, sulphonamides, tetracycline, trimethoprimsulfamethoxazole, chloramphenicol) detected in 19.6\% ESBL-producing E. coli isolates. No significant differences in resistance profiles and prevalence among isolates from sows, gilts and their piglets were observed (data not shown).

\section{Discussion}

The prophylactic and therapeutic use of antibiotics is known to be the cause of the high prevalence and long-term persistence of resistant strains of E. coli in food-producing 
Table 1. Excretion of ESBL-producing E. coli in faeces of positive sows and their offspring.

\begin{tabular}{|c|c|c|c|c|}
\hline \multirow{2}{*}{ Category } & \multirow{2}{*}{ No. of sow } & \multicolumn{2}{|c|}{ Counts in faeces after farrowing* } & \multirow{2}{*}{$\begin{array}{c}\text { Piglets } \\
\text { positive/examined }\end{array}$} \\
\hline & & Streak plating method & CFU/g faeces & \\
\hline & 1 & negative & $10^{1}$ & NT \\
\hline & 2 & $1+$ & $10^{1}$ & $6 / 11$ \\
\hline & 3 & $1+$ & $10^{2}$ & $10 / 10$ \\
\hline & 4 & $1+$ & $10^{1}$ & NT \\
\hline & 5 & $1+$ & $10^{1}$ & NT \\
\hline & 6 & $1+$ & nd & $0 / 8$ \\
\hline & 7 & $1+$ & $10^{2}$ & $1 / 8$ \\
\hline & 8 & $1+$ & $10^{2}$ & NT \\
\hline & 9 & $1+$ & $10^{2}$ & NT \\
\hline & 10 & $1+$ & $10^{3}$ & $2 / 3$ \\
\hline & 11 & $2+$ & $10^{3}$ & NT \\
\hline & 12 & $2+$ & $10^{2}$ & NT \\
\hline \multirow[t]{18}{*}{ Sows } & 13 & $1+$ & $10^{2}$ & $2 / 3$ \\
\hline & 14 & $1+$ & $10^{3}$ & $2 / 3$ \\
\hline & 15 & $2+$ & $10^{3}$ & $2 / 3$ \\
\hline & 16 & $2+$ & $10^{2}$ & $2 / 3$ \\
\hline & 17 & $1+$ & $10^{3}$ & NT \\
\hline & 18 & $2+$ & $10^{5}$ & $3 / 3$ \\
\hline & 19 & $4+$ & $10^{1}$ & $3 / 3$ \\
\hline & 20 & $1+$ & $10^{6}$ & $2 / 3$ \\
\hline & 21 & $4+$ & $10^{5}$ & $2 / 3$ \\
\hline & 22 & $4+$ & nd & $2 / 3$ \\
\hline & 23 & $3+$ & $10^{5}$ & $3 / 3$ \\
\hline & 24 & $4+$ & $10^{4}$ & $3 / 3$ \\
\hline & 25 & $3+$ & $10^{3}$ & NT \\
\hline & 1 & $3+$ & $10^{4}$ & NT \\
\hline & 2 & $3+$ & $10^{4}$ & $1 / 7$ \\
\hline & 3 & negative & $10^{3}$ & $5 / 6$ \\
\hline & 4 & $3+$ & $10^{4}$ & $4 / 6$ \\
\hline & 5 & $5+$ & $10^{4}$ & $2 / 6$ \\
\hline \multirow[t]{7}{*}{ Gilts } & 6 & $3+$ & $10^{4}$ & $5 / 5$ \\
\hline & 7 & $3+$ & $10^{4}$ & $5 / 5$ \\
\hline & 8 & $5+$ & $10^{5}$ & $5 / 5$ \\
\hline & 9 & $5+$ & $10^{3}$ & NT \\
\hline & 10 & $3+$ & $10^{3}$ & $5 / 5$ \\
\hline & 11 & $5+$ & $10^{5}$ & $5 / 5$ \\
\hline & 12 & $3+$ & $10^{4}$ & $5 / 5$ \\
\hline
\end{tabular}

*Bacterial growth on $\mathrm{MCA}_{\mathrm{CEF}}$ using the streak plating method was determined as follows: Growth of a few isolated colonies only in quadrant 1 was categorized as $1+$, growth of many colonies limited to quadrant 1 was categorized as 2+, growth limited to quadrants 1 and 2 was categorized. as $3+$, growth in quadrants 1,2 and 3 as $4+$ and bacterial growth that extended to all 4 quadrants was categorized as 5+; nd, not determined.

${ }^{+} \mathrm{NT}$, litter was not tested because of cross fostering piglets. 
Table 2. Resistance phenotypes to selected antimicrobials in ESBL-producing E. coli from pigs.

\begin{tabular}{|c|c|c|c|c|c|c|c|}
\hline \multirow{2}{*}{ Resistance phenotype } & \multirow{2}{*}{ No. of isolates } & \multicolumn{6}{|c|}{ Resistance to antimicrobials } \\
\hline & & $\mathrm{S}$ & S3 & TCY & SXT & CHL & NA \\
\hline 1 & 21 & & & & & & \\
\hline 2 & 16 & & & & & & \\
\hline 3 & 1 & & & & & & \\
\hline 4 & 2 & & & & & & \\
\hline 5 & 7 & & & & & & \\
\hline 6 & 1 & & & & & & \\
\hline 7 & 25 & & & & & & \\
\hline 8 & 10 & & & & & & \\
\hline 9 & 12 & & & & & & \\
\hline 10 & 2 & & & & & & \\
\hline 11 & 3 & & & & & & \\
\hline 12 & 1 & & & & & & \\
\hline 13 & 4 & & & & & & \\
\hline 14 & 1 & & & & & & \\
\hline 15 & 1 & & & & & & \\
\hline Total & 107 & 106 & 88 & 86 & 57 & 49 & 20 \\
\hline
\end{tabular}

Resistance is indicated by black boxes; S - streptomycin; S3 - suplhonamide compounds; TCY - tetracyclines; SXT - trimethoprim-suphamethoxazole; CHL - chloramphenicol; NA -nalidixic acid

animals. Various levels of ESBL producers have been documented in the pig production system mainly due to the differences in antibiotic policies on farms, including the use of higher generation cephalosporins. Cavaco et al. (2008) proved in their experimental study in pigs that cephalosporins (ceftiofur and cefquinom) have a high selective effect on ESBL-producing E. coli. In Denmark, ESBL-producing E. coli in pigs was detected on $79 \%$ of the farms with high consumption of cephalosporins compared to $20 \%$ on farms with no consumption of cephalosporins (Hammerum et al. 2014). In our study, ESBLproducing E. coli isolates were found in $25 \%$ sows and $34 \%$ gilts despite the fact that cephalosporins were banned on farm Amore than 24 months prior to the study. In Denmark, a significant effect was recognized on reduction of ESBL-producing $E$. coli prevalence on pig farms, in slaughtered pigs as well as pork meat approximately six months after the ban of cephalosporin use in pig production in the year of 2010 (Agersø et al. 2012; Agersø and Aarestrup 2013).

Similar to our study, other authors have also reported that the withdrawal of cephalosporins from pig farms did not lead to the immediate reduction of resistant $E$. coli in the respective herds (Andersen et al. 2015). High prevalence of CTX-M-producing E. coli was demonstrated in up to $71 \%$ sows and $97 \%$ piglets on Danish farms one year after the ban of cephalosporins (Hansen et al. 2013). However, these were farms with the history of high-level consumption of cephalosporins. It has been proposed that factors other than cephalosporins are likely involved in the persistence of ESBL producers on the farm. These may include the use of antibiotic from other groups, purchase of pigs from herds with history of cephalosporin use or contamination of feed, water and sludge by ESBLproducing isolates (Marshall and Levy 2011). Andersen et al. (2015) documented that the use of antimicrobials other than cephalosporins on pig farms is associated with higher probability of finding ESBL-producing E. coli. This association may be a result of co-selection by antimicrobials from other groups, since most of the isolates obtained 
from both sows and piglets in our study showed multi-resistance profiles. Resistances to tetracyclines and sulphonamides were the most common, including the combination with trimethoprim. These antimicrobials are indicated for the therapy of various bacterial infections in sows or piglets and may have resulted in co-selection and maintenance of ESBL-producing E. coli in the herd. Genes associated with ESBL phenotype in E. coli are often carried by transferrable plasmids along with genetic determinants conferring resistance to the above mentioned antimicrobials, therefore the use of multiple antimicrobial agents main impact the maintenance of ESBL plasmids (Carattoli 2013).

In contrast to other studies that were mainly focused on ESBL isolates in the intestine of slaughtered pigs, here we document ESBL-producing E. coli in a sow herd including their offspring. Our results indicate the circulation and long-term persistence of ESBLproducing $E$. coli in the herd even in the absence of selective pressure of cephalosporins. ESBL-producing isolates were likely selected in piglets treated by ceftiofur prior to the withdrawal followed by the transferand establishment in the gut microbiota of sows. Gilts originating from the multiplying herd on farm B with no history of cephalosporins were proved to be free of ESBL-producing isolates before their introduction to the herd on farm A. ESBL-producing isolates were found in rectal swabs of gilts three months after their introduction as well as in their faeces shortly after the farrowing. Our results indicate that sows of higher parity were the source of ESBL isolates not only for their offspring but also for the newly introduced gilts.

Interestingly, ESBL-producing E. coli were found in higher faecal densities in the introduced gilts compared to sows of second or higher parity. Moreover, most offspring of gilts and sows shed ESBL-producing E. coli, suggesting that the colonized dams and the farm environment contaminated by their faeces are plausible sources of these strains for the piglets. We assume that the colonization of the gut of new-born piglets by ESBL-producing E. coli happens shortly after the farrowing since these strains were found in the intestine of 12-hour-old offspring. Hansen et al. (2013, 2014) reported significant changes in carriage proportion, faecal counts and diversity of ESBL-producing E. coli in pigs during the production cycle. Reduction of prevalence of ESBL-producing E. coli accompanied by the decrease of mean faecal counts and strain diversity from piglets to finishers was observed. They suggested that observed differences reflect age-related physiological changes in the pig intestinal tract and as well as management procedures such as change in diet and antimicrobial treatment that are specific for each production stage.

In conclusion, results of this study showed long-term persistence of ESBL-producing E. coli in sow herd despite the ban of ceftiofur. Intestinal colonization of milking sows of the second and higher parity and their offspring as well as survivability of ESBL-producing E. coli in the farm environment should be recognised as important risk factors of their circulation and long-term persistence in pig production system.

\section{Acknowledgements}

We thank Jana Hofirkova for her assistance in the laboratory. The study was funded by CEVA and CEITEC 2020 - Central European Institute of Technology from European Regional Development Fund (LQ1601) from the Czech Ministry of Education, Youth and Sports within the National Programme for Sustainability II. Jan Vasek was supported by Internal Grant Agency of the University of Veterinary and Pharmaceutical Sciences Brno, Brno, Czech Republic (1042014/FVL).

\section{References}

Agersø Y, Aarestrup FM, Pedersen K, Seyfarth AM, Struve T, Hasman H 2012: Prevalence of extended-spectrum cephalosporinase (ESC)-producing Escherichia coli in Danish slaughter pigs and retail meat identified by selective enrichment and association with cephalosporin usage. J Antimicrob Chemother 67: 582-588 
Agersø Y, Aarestrup FM 2013: Voluntary ban on cephalosporin use in Danish pig production has effectively reduced extended-spectrum cephalosporinase-producing Escherichia coli in slaughter pigs. J Antimicrob Chemother 68: 569-572

Andersen VD, Andersen VD, Jensen VF, Vigre H, Andreasen M, Agersø Y 2015: The use of third and fourth generation cephalosporins affects the occurrence of extended-spectrum cephalosporinase-producing Escherichia coli in Danish pig herds. Vet J 204: 345-350

D’Andrea MM, Arena F, Pallecchi L, Rossolini GM 2013: CTX-M-type $\beta$-lactamases: a successful story of antibiotic resistance. Int J Med Microbiol 303: 305-317

Carattoli A 2013: Plasmids and the spread of resistance. Int J Med Microbiol 303: 298-304

Cavaco LM, Abatih E, Aarestrup FM, Guardabassi L 2008: Selection and persistence of CTX-M-producing Escherichia coli in the intestinal flora of pigs treated with amoxicilin, ceftiofur or cefchinome. Antimicrob Agents Chemother 52: 3612-3616

CLSI 2008: Clinical and Laboratory Standards Institute: Performance standards for antimicrobial disk and dilution susceptibility tests for bacteria isolated from animals, $3^{\text {rd }}$ edn. Approved standard. Document M31-A3. Clinical and Laboratory Standards Institute, Wayne, PA.

Denkel LA, Gastmeier P, Leistner R 2016: The association of ESBL-producing Enterobacteriaceae (ESBL-E) carriage in humans with pigs. Epidemiol Infect 144: 691-692

Dolejska M, Jurcickova Z, Literak I, Pokludova L, Bures J, Hera A, Kohoutova L, Smola J, Cizek A 2011: IncN

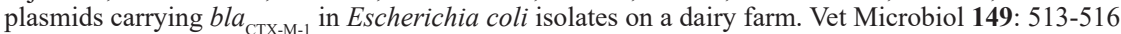

Dutil L, Irwin R, Finley R, Ng LK, Avery B, Boerlin P, Bourgault AM, Cole L, Daignault D, Desruisseau A, Demczuk W, Hoang L, Horsman GB, Ismail J, Jamieson F, Maki A, Pacagnella A, Pillai DR 2010: Ceftiofur resistance in Salmonella enterica serovar Heidelberg from chicken meat and humans, Canada. Emerg Infect Dis 16: 48-54

EFSA 2011: Panel on Biological Hazards (BIOHAZ). Scientific Opinion on the public health risks of bacterial strains producing extended-spectrum $\beta$-lactamases and/or AmpC $\beta$-lactamases in food and food-producing animals. EFSA Journal 9: 2322

EMA 2015: European Medicines Agency, European Surveillance of Veterinary Antimicrobial Consumption. Sales of veterinary antimicrobial agents in 26 EU/EEA countries in 2013. (EMA/387934/2015)

Hammerum AM, Larsen J, Andersen VD, Lester CH, Skovgaard Skytte TS, Hansen F, Olsen SS, Mordhorst H, Skov RL, Aarestrup FM, Agersø Y 2014: Characterization of extended-spectrum $\beta$-lactamase (ESBL)producing Escherichia coli obtained from Danish pigs, pig farmers and their families from farms with high or no consumption of third- or fourth-generation cephalosporins. J. AntimicrobChemother 69:2650-2657

Hansen KH, Bortolaia V, Damborg P, Guardabassi L 2014: Strain diversity of CTX-M-producing Enterobacteriaceae in individual pigs: insights into the dynamics of shedding during the production cycle. Appl Environ Microbiol 80: 6620-6626

Hansen KH, Damborg P, Andreasen M, Nielsen SS, Guardabassi L 2013: Carriage and fecal counts of CTX-Mproducing Escherichia coli in pigs: a longitudinal study. Appl Environ Microbiol 79: 794-798

Hornish RE, Kotarski SF 2002: Cephalosporins in veterinary medicine - ceftiofur use in food animals. Curr Top Med Chem 2: 717-731

Jørgensen CJ, Cavaco LM, Hasman H, Emborg HD, Guardabassi L 2007: Occurrence of CTX-M-1-producing Escherichia coli in pigs treated with ceftiofur. J Antimicrob Chemother 59: 1040-1042

Lutz EA, McCarty MJ, Mollenkopf DF, Funk JA, Gebreyes WA, Wittum TE 2011: Ceftiofur use in finishing swine barns and the recovery of fecal Escherichia coli or Salmonella spp. resistant to ceftriaxone. Foodborne Pathog Dis 8: 1229-1234

Marshall BM, Levy SB 2011: Food animals and antimicrobials: Impacts on human health. Clin Microbiol Rev 24: 718-733

Pitout JD, Laupland KB 2008: Extended-spectrum beta-lactamase-producing Enterobacteriaceae: an emerging public-health concern. Lancet Infect Dis 8: 159-166

Seiffert SN, Hilty M, Perreten V, Endimiani A 2013: Extended-spectrum cephalosporin-resistant Gram-negative organisms in livestock: an emerging problem for human health? Drug Resist Updat 16: 22-45

Smet A, Martel A, Persoons D, Dewulf J, Heyndrickx M, Herman L, Haesebrouck F, Butaye P 2010: Broadspectrum $\beta$-lactamases among Enterobacteriaceae of animal origin: molecular aspects, mobility and impact on public health. FEMS Microbiol Rev 34: 295-316

Tragesser LA, Wittum TE, Funk JA, Winokur PL, Rajala-Schultz PJ 2006: Association between ceftiofur use and isolation of Escherichia coli with reduced susceptibility to ceftriaxone from fecal samples of dairy cows. Am J Vet Res 67: 1696-1700

Van Saene HFK, Izquierdo G, Garcia-Hierro P, Fontana F 2012: Gut Microbiology: Surveillance Samples for the Detection of the Abnormal Carrier State, Chapter 6, p. 75-87, In: Van Saene HKF, Silvestri L, de la Cal MA, Gullo A (Eds): Infection Control in the Intensive Care Unit. Springer-Verlag Italia, 513 p. 